\title{
Effect of Caffeine on Multi-domain Neurocognitive Abilities in Occasional Coffee Consumers
}

\author{
Sasikala P. ${ }^{1}$, A.V. Siva Kumar, ${ }^{2}$, K.N.Maruthy ${ }^{3}$, John Preetham kumar Gurja ${ }^{4}$ \\ ${ }^{1}$ Professor, ${ }^{2}$ Ph.D Scholar, ${ }^{3}$ Professor \& Head, ${ }^{4}$ Assistant Professor, Dept. of Physiology, Narayana Medical \\ College and Hospital, Nellore Andhra Pradesh, India
}

\begin{abstract}
Background: Multi-domain neurocognitive abilities underpin timely perception \& appropriate action to unexpected threats in the dynamic environment. Hence enhancement of the same may be life-saving in some situations. Caffeine is the most widely consumed CNS stimulant which not only enhances neurocognitive abilities but also temporarily counteracts neurocognitive deficits. Therefore this study has been undertaken to quantify the effect of Caffeine on Multi-domain neurocognitive skills.
\end{abstract}

Aim: To find out the effect of Caffeine on neurocognitive abilities in occasional coffee consumers

Materials \& Methods: This study was conducted on 23 participants. In all the subjects under basal conditions, a battery of multi-domain neurocognitive tests, which included ART, VRT, CFFF, Digits forward tests were conducted. Then a standard dose of 2.2 grams of instant coffee powder (Nestle $-69.6 \mathrm{mg}$ of Caffeine) dissolved in $100 \mathrm{ml}$ of milk was given. After 30 minutes of intake, the same battery of tests was repeated, and the test results were analyzed.

Results: In our study, we have found that Caffeine significantly increases CFFF ( $\mathrm{p}<0.001$ ), Digits forward test score $(\mathrm{p}<0.001)$ and decreases VRT $(\mathrm{p}=0.03)$. It also reduces ART, but it is not statistically significant. $(\mathrm{p}=0.06)$.

Conclusion: Caffeine significantly improves Critical flicker fusion frequency, attention span, performance and reduces reaction time.

Keywords: Caffeine, Auditory Reaction Time, Visual Reaction Time, Critical Flicker Fusion Frequency, Digits forward test.

\section{Introduction}

The mission of National safety council is to eliminate preventable deaths at work, in homes, communities and on roads through Leadership, Research, Education and Advocacy. Drowsiness, inattention \& impaired neurocognitive abilities are among the public safety hazards which risks a person's as well as public life. Impaired neurocognitive skills are not only dangerous at home, but also the workplace and on-road. At the workplace, safety performance decreases as employees become tired; $62 \%$ of night-shift workers complain about sleep loss.Fatigued worker productivity costs employers $\$ 1,200$ to $\$ 3,100$ per employee annually. Employees on rotating shifts are particularly vulnerable because they cannot adapt their "body clocks" to an alternative sleep pattern ${ }^{1}$. Coming to road safety, National Highway Traffic Safety Administration estimates, drowsy driving was responsible for 72000 crashes 44,000 injuries, and 800 deaths in $2013^{2}$. However, these numbers are underestimated, and up to 6000 fatal crashes, each year may be caused by drowsy drivers. Cognition reflects the processes by which sensory input is transformed, reduced, elaborated, stored, recovered $\&$ used. The domains of cognition include attention, executive function, memory, visuospatial function and language ${ }^{3}$. These multi-domain neurocognitive abilities underpin timely perception \& appropriate reaction to unexpected threats in the dynamic environment. Hence 
enhancement of the same may be life-saving in some situations. Caffeine because of itspsychostimulant properties, easy availability, legal and social acceptance, low toxicity and abuse potential may be considered for enhancement of multi-domain neurocognitive abilities ${ }^{4}$. Hence this project has been undertaken to study the effect of caffeine on Multifield neurocognitive abilities in Occasional Coffee consumers.

\section{Materials \& Methods}

Study population: It is a quasi-experimental [Interventional] study, conducted on 23 subjects, out of which eight subjects were females, and 15 were males. The subjects who have acquaintance to the investigators were recruited using a non-randomized sampling technique. The subjects were inquired about the history of medical illness, their lifestyle andbehaviour. Following anthropometric measurements, they were subjected to a battery of Neurocognitive function tests, which includes:

\section{Auditory Reaction time \& Visual Reaction time:}

Auditory Reaction time \& Visual Reaction time were recorded using an in house built device called PC1000. It is described as $1000 \mathrm{Hzsquare}$ wave generator \& Module A \& B with which we start and stop the oscillator. Module A has Start button \& Module B has a stop button.Auditory Reaction Time, Subject is instructed to press the stop button in module B when he hears a tone of $1000 \mathrm{~Hz}$ in headphones. The number of oscillations produced in this period recorded and reported as the Auditory Reaction Time with an accuracy of $1 \mathrm{msec}$. For determining Visual Reaction Time, the same device had been used, and the only difference is the subject is provided with a visual stimulus, a red colour light which is $5 \mathrm{~mm}$ in size (LED- Light Emitting Diode). In module A when the start button is pressed, the red light glow in module $\mathrm{B}$, for which the subject is instructed to press the Stop button in module B. The numberOf Oscillations produced by the oscillator in this period is recorded as the Visual Reaction Time with an accuracy of $1 \mathrm{msec}^{5,6}$.

\section{Critical Flicker Fusion Frequency:}

CFFF was measured using an in house built device which can produce a Red light-emitting diode of $5 \mathrm{~mm}$ size at frequencies in the range of $10 \mathrm{~Hz}$ to $60 \mathrm{~Hz}$ (Square wave) with the help of a software called as "Sweepgen".
The participants sit in front of the module at a distance of $25-30 \mathrm{~cm}$ in a less illuminatedroom. The Red light is made to flicker at a low frequency of $10 \mathrm{~Hz}$. The subject is instructed to prompt when the flickering stops. In the next step, frequency is increased gradually at a rate of $1 \mathrm{~Hz}$ at a time. The frequency at which the subject is no longer able to discriminate individual flickers and perceives it as asingle stimulus is reported as $\mathrm{CFFF}^{7,8}$.

\section{Digits forward test:}

In psychology and neuroscience, memory span is the most extended list of items that a person can repeat back in correct order immediately after presentation,i.e., it is a measure of working memory. In Digits span test, a series of random numbers in the ascending order from 1 to 10 are told to the subject and each time he is asked to repeat the set of numbers in the correct order. Digit Span tests your ability to remember a sequence of numbers that are told, one at a time, in the same order. If you correctly recall all of the numbers, then the next serieswas one number longer. If you make a mistake, then the following sequence was one number shorter. the test ends after three mistakes,. In this test accuracy does matter; after three errors, the trial ends. However, wrong answers do not subtract from thescore of the subject, which is the maximum number of digits that are correctly repeated.

\section{Intervention:}

Standardization of coffee: According to USDA (United States Department of Agriculture) 1.8 grams of Nestle Instant coffee powder comprises of $57 \mathrm{mg}$ of caffeine. Therefore the regular 2.2 grams of Nestle coffee powder sachets consists of $69.66 \mathrm{mg}$ of caffeine. So in our study, we have used 2.2 grams of Nestle coffee powder of same batch comprising of $69.66 \mathrm{mg}$ of caffeine dissolved in $100 \mathrm{ml}$ of milk to study effects of caffeine on multi-domain neurocognitive abilities ${ }^{9}$.

After conducting the above mentioned multi-domain neurocognitive teststo each subject, a standard dose of 2.2grams of instant coffee powder of same batch (Nestle - 69.6mg of caffeine) dissolved in $100 \mathrm{ml}$ of milk was given. After 30 minutes of intake, the same battery of tests was repeated, and the test results were analyzed\& the effect of caffeine on temporalcognition was studied and quantified. 


\section{Statistical Analysis}

The data was analyzed by using Graph pad prism (Trail version). The information was expressed as the Mean \pm standard deviation. The data were subjected to find outnormal Gaussian distribution using the Kolmogorov - Smirnov test. As the data sets were skewed, Non-parametric test (Man Whitney - U test) was performed to find out the differences between before and after the intervention. The correlation coefficient ( $\mathrm{r}$ ) was analyzed using the Spearman correlation to determine associations. A p-value $<0.05$ was considered as statistical significant.

\section{Results}

The demographic features of the subjects were shown in table -1 . The mean age of the participantswas 29.19 \pm 7.62 years with Confidence Interval $(26.11-32.27$ years). The mean height and weights were 157.8 $\pm 12.77 \mathrm{~cm}$ and $62.23 \pm 11.56 \mathrm{~kg}$, respectively. The BMI was calculated using the conventional formula, and the mean BMI was $25 \pm 4.1 \mathrm{~kg} / \mathrm{m}^{2}$. The effect of caffeine was analyzed with an interval of $30 \mathrm{~min}$. The different neurocognitive parameters were measured using simple reaction times and Critical flicker Fusion Frequency,which were shown in Table no - 2. We have observed a statisticallysignificant difference before and after intervention with caffeine in CFFF (p-0.001), $\operatorname{VRT}(p-0.05)$ and DigitS forward test (p-0.001). There was no statistical significance associated withART(pvalue - 0.06). However, there was a trend showing a decline in ART on intervention with caffeine.

Table1: Shows Demographic features

\begin{tabular}{|l|l|l|l|}
\hline Demographic features & Mean \pm SD & Median & $\mathbf{9 5 \%}$ CI \\
\hline Age (yrs) & $29.19 \pm 7.62$ & 23.75 & $26.11-32.27$ \\
\hline Weight $(\mathrm{kg})$ & $62.23 \pm 11.56$ & 60 & $57.56-66.9$ \\
\hline Height $(\mathrm{cm})$ & $157.8 \pm 12.77$ & 159 & $152.7-163$ \\
\hline BMI $(\mathrm{kg} / \mathrm{m} 2)$ & $25 \pm 4.1$ & 24.05 & $23.35-26.67$ \\
\hline
\end{tabular}

Table 2 shows Multi domain neuro cognitive parameters before and after intervention with caffeine

\begin{tabular}{|l|l|l|l|}
\hline Neurocognitive tests & Before & After & P value \\
\hline ART & $148.4 \pm 25.05$ & $139.5 \pm 25.53$ & 0.06 \\
\hline VRT & $197.7 \pm 30.4$ & $180.2 \pm 25.05$ & $<0.05$ \\
\hline CFFF & $41.58 \pm 3.7$ & $43.27 \pm 3$ & $<0.001^{* * *}$ \\
\hline DFT & $7.11 \pm 2$ & $7.82 \pm 2.1$ & $<0.001^{* * *}$ \\
\hline
\end{tabular}




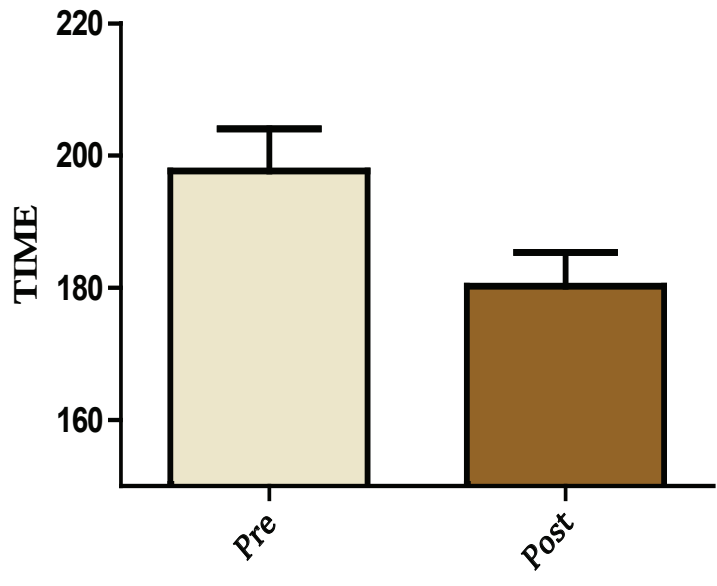

VRT

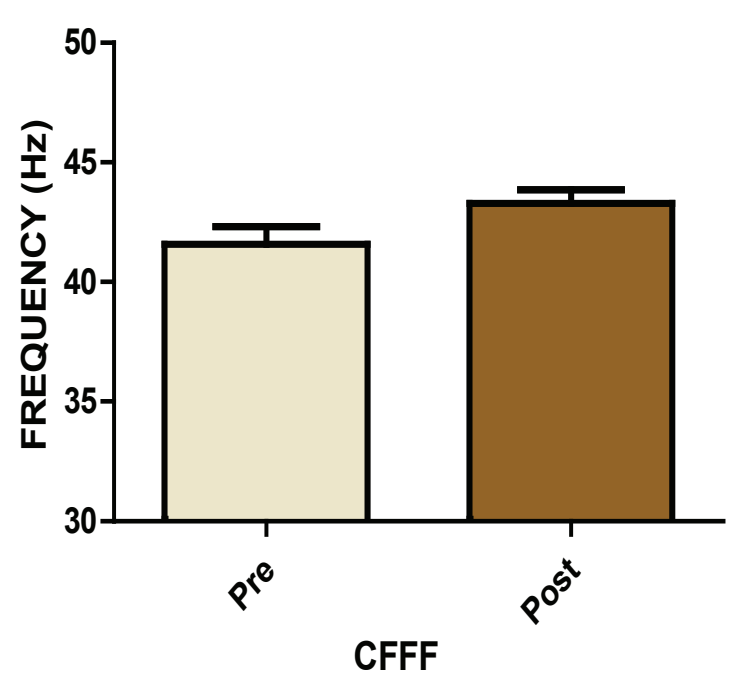

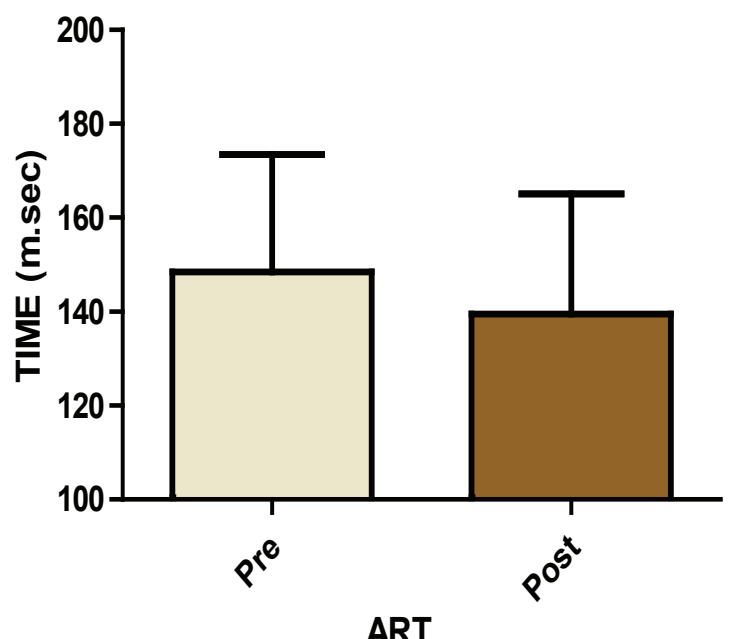

ART

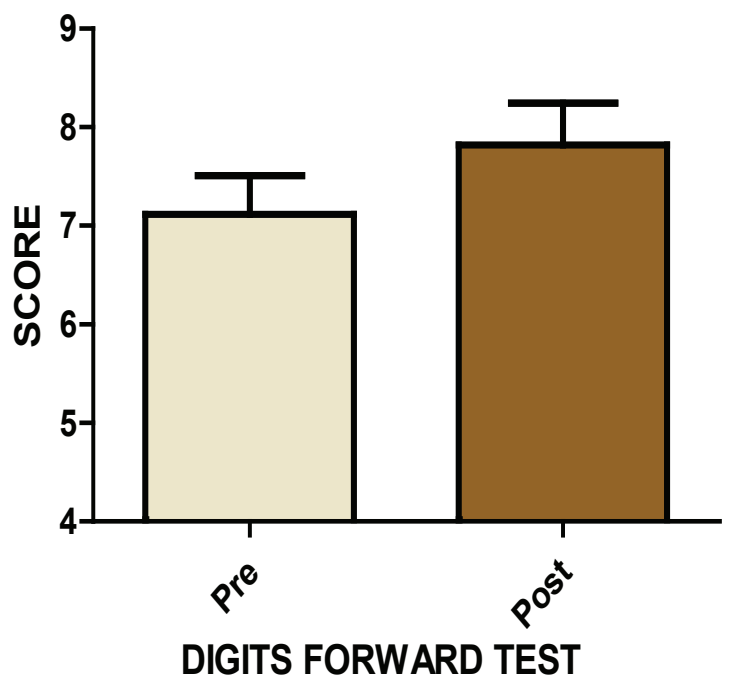

Figure1 shows the differences of multi domain neuro cognitive parameters before and after caffeine intervention

\section{Discussion}

Natural and humanmade disasters can strike anywhere and at any time. So it is essential to have a planned response at home, at work and on-road. Inattentiveness \&inability to predict the hazards sometimes costs life at home. At the workplace, the National Safety Council says that Safety performance decreases as employees become tired.62\% of night-shift workers complain about sleep loss.Fatigued worker productivity costs employers $\$ 1,200$ to $\$ 3,100$ per employee annually.Employees on rotating shifts are particularly vulnerable because they cannot adapt their "body clocks" to an alternative sleep pattern". Similarly, on-road, a drowsy driver is three times more likely to be in a car crash. Driver's Reaction times, awareness of hazards and ability to sustain attention all worsen when the driver is drowsy. Inattentiveness \& inability to predict the risks sometimes costs life even at home. Toprevent these mishaps, National Safety Council has suggested some interventions to avoid drowsy driving, i.e., Crash avoidance technologies, University interventions, promoting 7hours of sleep each night, Medication labels for drugs causing drowsiness, Health programmers for employees regarding the importance of sufficient sleep.Our study adds a readily available, economically 
affordable, socially acceptable intervention to the list to drive away drowsiness and fatigue ${ }^{17}$. Alertness $\&$ arousal are maintained by Ascending Reticular Activating System. These projections fire in a characteristic pattern to promote arousal. However, every 24 hours, during a sleep arousal system is inhibited by sleep-active $\gamma$-aminobutyric acid (GABA)-ergic and galaninergic neurons of the ventrolateralpreoptic nucleus (VLPO). The interaction between the VLPO and the branches of the ascending arousal pathway is mutually inhibiting, functioning much like an electrical «on-off» switch, enabling the body to maintain a stable state of wakefulness and sleep. When the brain does not get enough sleep ATP is co-released with neurotransmitters. Adenosine is the secondary bye product ofATP. During prolonged alertness, physical and mental fatigue, the extracellular concentration of Adenosine increasesin the Cortex and Basal forebrain. Adenosine exerts it» s somnergic effects by binding to it» $\mathrm{s}$ receptors-i.e., A1, A2A. The underlying mechanisms for the sleepinducing effects of Adenosine are 1. Slowing down the neuronal activity 2. Dilatation of blood vessels supplying the brain to let more Oxygen into that organ during sleep. Caffeine, the world's most widely consumed psychoactive drug \& central nervous system (CNS) stimulant of the methylxanthine class ${ }^{16}$. Caffeine binds to Adenosine receptors, i.e., $\mathrm{A}_{1}, \mathrm{~A}_{2 \mathrm{~A} \text { in }}$ the central nervous system (CNS) and inhibits Adenosine from binding. Thus hindering the Adenosine mediateddownregulation of CNS activity and by promoting central dopamine \&noradrenaline transmission ${ }^{11}$.

In a study conducted by, Usha G. Shenoy et al., "The Influence of Caffeine on the Visual and Auditory Reaction Time in Medical Students "the authors concluded that Visual reaction time \& Auditory reaction time were significantly reduced after the consumption of caffeine $(p<0.05)$. Therefore caffeine is a potent stimulant of the Central nervous system. In our study, we have found that though caffeine reduces both Auditory as well as Visual reaction time the reduction in the visual reaction time is found to be statistically significant $(p=0.03)$ The three main mechanisms involved in the decrease in reaction time by Caffeine are: $1^{10}$. It improves physical performance and mental alertness due to the neurohormonal influence of caffeine which helps in mobilization of the intracellular calcium, and it inhibits an enzyme called phosphodiesterase, which breaks cAMP and increases cellular activity resulting in generalized stimulation. The only likely mechanism of action of methylxanthine is antagonism at the level of adenosine receptors. Caffeine increases metabolism throughout the brain but at the same time decreases the blood flow, including relative brain hypoperfusion. In a study conducted by GromeJJ et al. it has been concluded that caffeine increases local cerebral glucose utilization and decreases regional cerebral blood flow. Therefore it resets the ratio of cerebral blood flow Glucose utilization at a lower level. Besides, It activates noradrenaline neurons and seems to increase the local release of dopamine $^{14,15}$.

CFFF is one of the best means to measure cortical arousal. It is defined as the frequency at which an intermittent light stimulus appears to be entirely steady for the average human observer. Owen GN et al. stated that caffeine improvises the Cognitive performance demanding tasks associated with rapid visual information processing, critical flicker fusion threshold, attention switching and mood. Quantitatively caffeine improved subjective alertness at $60 \mathrm{~min}$ and accuracy on the attention-switching task at $90 \mathrm{~min}$ period of post Coffee consumption $^{12}$.

Digits forward test is a measure of working memory, i.e., short term auditory memory. In a study conducted by Stephanie M. Sherman, Timothy P. Buckley, ElsaBaena et al. stated "Caffeine Enhances Memory Performance in Young Adults during their Non-optimal Time of Day $^{13}$. Theauthors of the same study have concluded thatcaffeine has a specific benefit for memory during student's non-optimal time of day - early morning. The current research also shows a statistically significant increase in the score was noted on Digits forward test. Koppelsletter et al.studied about a dozen healthy adults and reported that caffeine boosts activity in the brain regions related to attention and short-term memory. There are recent advanced techniques to quantify the temporal cognition which are user friendly and cost effective ${ }^{18,19,20}$.

Limitations: The current study has a few limitations. The results of the present study cannot be generalized to the population because of the lesser sample size and recruitment of subjects by convenient sampling technique. The long term effects of caffeine were not 
studied.The cognition is a large domain which cannot be attributed by assessing choice and exact reaction times alone.We are planning to extend our study design with more sample size and subjects will be followed up to find out thelong term effects of caffeine on multi-domain neurocognitive abilities.

\section{Conclusion}

From the study entitled "Effect of Caffeine on Multi-domain neurocognitive abilities" it has been concluded that intervention with caffeine significantly improves Critical flicker fusion frequency, attention span, performance and reduces Simple reaction times like Visual \& Auditory reaction times.

Implications: The current study provides newer insights for reliable, practicable, safer andinstantaneous intervention to drive away drowsiness. It also reduces reaction time and improves the ability to sustain attention, to eliminate preventable deaths in homes, on roads and at workplaces.

Ethical Considerations: The study was reviewed and approved by the Institutional Ethics Committee of Host institution.

Funding: There was no financial support for conducting the study and publication of the article.

Conflict of Interest:On behalf of all the authors, corresponding authors states that there is no potential conflict of interest to publish this article.

Acknowledgements: Authors would like to express their sincere gratitude to the participants of the study for their cooperation during data acquisition. The authors are thankful to the Management of Narayana Medical College and Hospital for their constant support during the entire study period.

\section{References}

1. The National Safety Council,https://www.nsc.org/.

2. Sinha O, Singh S, Mitra A, Ghosh SK, Raha S. Development of a drowsy driver detection system based on EEG and IR-based eye blink detection analysis. Advances in Communication, Devices and Networking 2018 (pp. 313-319). Springer, Singapore.
3. Barnard PJ, Teasdale JD. Interacting cognitive subsystems: A systemic approach to cognitiveaffective interaction and change. Cognition \& Emotion. 1991 Jan 1;5(1):1-39.

4. Tully J, Montgomery C, Maier LJ, Sumnall HR. 9 Estimated prevalence, effects and potential risks of substances used for cognitive enhancement. Human Enhancement Drugs. 2019 Jun 12.

5. Sasikala P, MaruthyK N, Deepthi Sahithi T, "Assessment of Cognition and Psychomotor Skills in Anaemic Patients". International Journal of Physiology.2017 Jan 1;5(1):131-134.

6. Kumar AP, Kumar KM, Padmavathi R. Validation of PC $1000 \mathrm{~Hz}$ Reaction Timer with Biopac ${ }^{\circledR}$ MP 36 for Recording Simple Reaction Time. Indian J PhysiolPharmacol. 2019;63(2):138-44.

7. Kumar CK, Kumar AV, Madhurima P, Maruthy KN, Preetham GJ. Assessment of psychomotor skills using finger pulse guided biofeedback tool in young medical students. Annals of Medical Physiology. 2018 Dec 31.

8. Sasikala P, Maruthy K.N., Kumar CH. Kiran." Effect of Body Fat Percentage on Cognition in Males \&Females".International Journal of Physiology.2018;6(2):95-98.

9. Vega FE, Rosenquist E. The coffee berry borer and coffee research at the United States Department of Agriculture. InProc. First ICO World Coffee Conference, London, UK 2001.

10. Usha G. Shenoy ,Vinutha Shankar „NachalAnnamalai," The Influence of Caffeine on the Visual and Auditory Reaction Time in Medical Students", IOSR Journal of Dental and Medical Sciences (IOSR-JDMS) e-ISSN: 2279-0853, p-ISSN: 2279-0861.Volume 17, Issue 5 Ver. 4 (May. 2018), 46-49

11. Nehlig A, Daval JL, DebryG.Caffeine and the central nervous system: mechanisms of action, biochemical, metabolic and psychostimulant effects. Brain Research Reviews. 1992 May 1;17(2):139-70.

12. Owen GN, Parnell H,De Bruin EA,RycroftJA. The combined effects of L-theanine and caffeine on cognitive performance and mood.Nutritional neuroscience.2008 Aug 1;11(4):193-8. 
13. Stephanie M. Sherman, Timothy P. Buckley, ElsaBaena and Lee Ryan "Caffeine Enhances Memory Performance in Young Adults during Their Non-optimal Time of Day.Front Psychol.2016;7:1764.

14. GromeJJ,Stefanovich V. "Differential effects of methylxanthines on local cerebral blood flow and glucose utilization in the conscious rat". Pharmaco1986 Jun;333(2):172-178.

15. Dixit A, Goyal A, Thawani R, Vaney N. Effect of caffeine on information processing: evidence from stroop task. Indian journal of psychological medicine. $2012 \mathrm{Jul} ; 34(3): 218$.

16. Bruns, R.F., Adenosine and xanthines. In V. Stefanovich and I. Okyayuz-Baklouti (Eds.), Role of Adenosine in Cerebral Metabolism and Blood Flow, VNU Science Press, Utrecht, 1987, pp. 5780 .
17. Jonathan R.L Schwartz and Thomas Roth," Neurophysiology of Sleep and Wakefulness: Basic Science and Clinical Implications". CurrNeuropharmacol. 2008 Dec; 6(4): 367-378.

18. Kumar AS, Padmavathi R, Maruthy KN, Sowjanya B, Kumar K. An Innovative Technique to Evaluate Quantitative Pupillary Light Reflex by Dynamic Pupillometry using Infrared Videography. Journal of Clinical \& Diagnostic Research. 2019 Apr $1 ; 13(4)$.

19. Kumar CK, Maruthy KN, Sasikala P, Gurja JP, Kumar AV, Kareem SK. Impact of chronic alcoholism on temporal cognition and coordination of motor activity. International Journal of Physiology. 2018;6(4):124-7.

20. Ramadevi P, Maruthy KN. Evaluation of static pupil size using digital web camera through image analysis. International Journal of Physiology. 2019 Jul 25;7(3):7-11. 